Revue internationale P.M.E.

Économie et gestion de la petite et moyenne entreprise

\title{
Développement international de la très petite entreprise dans la région de Girona
}

\section{Anna Ronquillo et Carme Saurina}

Volume 7, numéro 2, 1994

URI : https://id.erudit.org/iderudit/1008392ar

DOI : https://doi.org/10.7202/1008392ar

Aller au sommaire du numéro

\section{Éditeur(s)}

Presses de l’Université du Québec

ISSN

0776-5436 (imprimé)

1918-9699 (numérique)

Découvrir la revue

Citer cette note

Ronquillo, A. \& Saurina, C. (1994). Développement international de la très petite entreprise dans la région de Girona. Revue internationale P.M.E., 7(2), 117-136. https://doi.org/10.7202/1008392ar

\section{Résumé de l'article}

Cette analyse sur l'internationalisation de la très petite entreprise (TPE) étudie ce phénomène dans les cantons de Girona. Il s'agit d'une région présentant les caractéristiques socio-économiques pour réaliser une telle étude : un grand nombre de petites entreprises et un niveau socio-économique élevé. Nous avons utilisé un questionnaire permettant de trouver les variables qui peuvent affecter le niveau de développement international des entreprises. Les résultats indiquent que l'attitude du chef d'entreprise, ainsi que les caractéristiques générales ou structurelles de l'entreprise sont les facteurs les plus importants en ce qui concerne le succès de l'activité internationale. Les entreprises analysées ont été classées en trois groupes : celles qui jouent un rôle influent sur leur environnement, avec un esprit entreprenant et une attitude combative non seulement en ce qui concerne la recherche de l'information nécessaire permettant de réaliser les exportations, mais aussi le dépassement des difficultés réelles de l'exportation tout en ayant une vision optimiste du développement de la crise actuelle; celles qui se laissent influencer par leur environnement avec des attitudes moins dynamiques et une plus grande préoccupation quant à leur futur immédiat; enfin, les entreprises traditionnelles avec des attitudes réservées quant à leur implication internationale.
Ce document est protégé par la loi sur le droit d'auteur. L'utilisation des services d'Érudit (y compris la reproduction) est assujettie à sa politique d'utilisation que vous pouvez consulter en ligne.

https://apropos.erudit.org/fr/usagers/politique-dutilisation/ 


\title{
Développement international de la très petite entreprise dans la région de Girona
}

\author{
Anna RONQUILLO * \\ Carme SAURINA ** \\ Université de Girona
}

\begin{abstract}
RÉSUMÉ
Cette analyse sur l'internationalisation de la très petite entreprise (TPE) étudie ce phénomène dans les cantons de Girona. Il s'agit d'une région présentant les caractéristiques socio-économiques pour réaliser une telle étude: un grand nombre de petites entreprises et un niveau socio-économique élevé. Nous avons utilisé un questionnaire permettant de trouver les variables qui peuvent affecter le niveau de développement international des entreprises. Les résultats indiquent que l'attitude du chef d'entreprise, ainsi que les caractéristiques générales ou structurelles de l'entreprise sont les facteurs les plus importants en ce qui concerne le succès de l'activité internationale. Les entreprises analysées ont été classées en trois groupes: celles qui jouent un rôle influent sur leur environnement, avec un esprit entreprenant et une attitude combative non seulement en ce qui concerne la recherche de l'information nécessaire permettant de réaliser les exportations, mais aussi le dépassement des difficultés réelles de l'exportation tout en ayant une vision optimiste du développement de la crise actuelle; celles qui se laissent influencer par leur environnement avec des attitudes moins dynamiques et une plus grande préoccupation quant à leur futur immédiat; enfin, les entreprises traditionnelles avec des attitudes réservées quant à leur implication internationale.
\end{abstract}

* Anna Ronquillo est licenciée ès sciences économiques et de l'entreprise et ès psychologie. Elle est professeure titulaire du Département d'économie de la Faculté des sciences juridico-économiques de l'Université de Girona. Publication : «Impact of the Olympic Games on the Economy of Catalonia », International Review for Business Education, Odense, 1993.

** Carme Saurina est licenciée ès sciences physiques. Diplôme de $3^{\mathrm{e}}$ cycle en statistiques appliquées. Elle est professeure titulaire du Département d'économie de la Faculté des sciences juridico-économiques de l'Université de Girona.

Adresse: Université de Girona, Faculté des sciences juridico-économiques, Département d'économie, Plaça Sant Domènec, 9, 17071 Girona, Espagne. 


\begin{abstract}
This analysis of the international development of the very small enterprise has been made in the cantons of Girona because this region has the appropirate social and economic characteristics for making this type of study: a great number of very small firms and a very high level of social and economic development. A questionnaire was used to find the variables that can affect the level of international development of the firms. The results indicate that the attitude of the owner or manager and some of the general characteristics of the firm are the most important success factors in the international activity. The firms analysed can be classified in three groups: the ones that influence their surroundings with an enterprising spirit and an aggressive attitude towards finding the necessary information for exporting as well as for getting through the actual difficulties that this activity presents, the ones that let the surroundings influence them, with less aggressive attitudes and higher worries about their immediate future, and the traditional ones, with more reserved attitudes towards their international projection.
\end{abstract}

\title{
RESUMEN
}

Este análisis sobre la internacionalización de la muy pequeña empresa se realiza en las comarcas de Girona por ser una región que tiene las características socio-económicas necesarias para la realización de este tipo de estudio: Un gran número de pequeñas empresas y un elevado nivel socioeconómico. Se utiliza un cuestionario para encontrar las variables que pueden afectar el nivel de desarrollo internacional de las empresas y los resultados indican que la actitud del empresario es el factor más importante para tener éxito en la actividad internacional. Las empresas analizadas quedan clasificadas en tres grupos: Las que inciden sobre su entorno con un espíritu emprendedor y una actitud agresiva tanto respecto a la búsqueda de la información necesaria para llevar a término las exportaciones, como sobre la superación de las dificultades reales que presenta la exportación y con una visión esperanzada sobre el desarrollo de la crisis actual, las que se dejan influir por su entorno con actitudes menos agresivas y con una mayor preocupación respecto a su futuro inmediato, y las tradicionales con actitudes más retraídas respecto a su proyección internacional.

\section{Introduction}

L'internationalisation croissante des marchés provoque l'augmentation de la concurrence potentielle pour les petites entreprises, mais leur offre également certaines possibilités d'agrandir leur marché grâce aux marchés extérieurs et de disposer également d'économies d'échelle.

Les très petites entreprises rencontrent de très grandes difficultés quand elles veulent exporter à cause du manque de capacités de production et du manque de connaissances concernant les marchés des autres pays, ainsi que les démarches à suivre afin de pénétrer les marchés étrangers : dispositions légales, administratives, etc. 
Aussi, notre recherche a-t-elle pour but d'étudier la problématique des très petites entreprises (TPE) des cantons de Girona, une région qui, grâce à ses caractéristiques socio-économiques et frontalières avec la France, semble une région idéale pour ce type d'analyse.

Notre étude a un double objectif. D'une part, elle essaie de trouver les variables les plus significatives permettant de caractériser, en général, les entreprises qui ont réussi leur internationalisation. D'autre part, elle définit des typologies concrètes des très petites entreprises exportatrices des cantons géronais.

Pour ce faire, nous avons d'abord effectué une analyse générale de la situation économique des entreprises de Girona. Puis, nous avons effectué une étude empirique pour mesurer les facteurs techniques et humains des entreprises en cherchant les caractéristiques conditionnant leur succès à l'exportation des produits.

\section{Caractéristiques de la région de Girona}

Les cantons de Girona présentent certaines conditions qui devraient normalement favoriser l'exportation. D'abord, la situation géographique. La situation frontalière de Girona avec la France fait que les possibilités d'internationaliser l'entreprise par l'achat ou la vente dans ce pays sont nombreuses. La proximité de marchés extérieurs constitue une variable très importante pour exporter ou importer et ce, pour deux raisons :

1. Les coûts de transport. Girona est aussi près de la région française du Languedoc-Roussillon que du reste de la région catalane à laquelle elle appartient. Vendre en France entraîne les mêmes coûts de transport que dans le reste de la Catalogne. L'entrée dans la CE et la suppression des barrières douanières facilitent encore l'accès au marché français.

2. La ressemblance des goûts. La proximité avec la France favorise une ressemblance de goûts créant une demande sous-jacente, une condition nécessaire à l'existence d'un commerce interbranche (Linder 1961) ou de produits similaires entre pays de développement similaire.

Les caractéristiques socio-économiques des cantons de Girona. La population de Girona est d'environ 500000 habitants. La population est encore jeune, tout en présentant une certaine tendance au vieillissement. Le taux d'activité (population active/total de la population entre 16 et 65 ans, soit l'indicateur de la structure productive et du dynamisme économique) est le plus élevé de l'Espagne $(68,1 \%)$. Le niveau de revenu est aussi le plus élevé. 
En retour, le taux de chômage est particulièrement bas, $7,2 \%$. Soulignons aussi que le pourcentage de jeunes qui cherchent un premier emploi est très faible. de TPE ${ }^{1}$.

Un nombre élevé de TPE. On trouve 11851 entreprises dont $88,5 \%$

La structure des entreprises. Le dynamisme d'une zone déterminée est en rapport avec la structure des entreprises de cette zone. Une large base d'entreprises localement contrôlée est le signe d'un bon esprit entrepreneurial autochtone (Backman, 1983). De plus, la présence de capital étranger permet de penser que la zone présente suffisamment de centres d'intérêt pour que cela favorise l'implantation d'entreprises. Girona réunit ces deux conditions. On trouve, d'une part, un grand nombre de petites et de moyennes entreprises et, d'autre part, des investissements étrangers dans un certain nombre de ces entreprises.

C'est dans les branches du textile, du papier et de la chimie qu'apparaissent les plus grandes entreprises contrôlées par des capitaux nationaux ou étrangers ; par contre, dans le secteur de l'alimentation, du liège, du bois et de la métallurgie, les petites entreprises à capital local sont les plus importantes.

\section{Cadre théorique}

Au cours des vingt dernières années, on a réalisé de nombreuses études sur l'internationalisation des PME/PMI. On peut diviser ces études en deux grands groupes en fonction du type d'analyse qu'elles réalisent:

1. Celles qui insistent sur l'analyse des facteurs intervenant sur la décision de devenir international ou non (Wiedersheim et al., 1978; Roux, 1986 et 1991; Reid, 1981; Garnier, 1982; Higler et Hoover, 1989).

2. Celles qui cherchent à expliquer le niveau de succès de cette internationalisation, une fois prise la décision d'exporter. Une première synthèse de ces travaux a été réalisée par Bilkey (1978). On doit citer aussi celles de Baudoux et Roncin (1980), Reffait et Roux (1981), Pras et Roux (1985) et Roux (1991), dans lesquelles on précise les facteurs suivants : les caractéristiques générales de l'entreprise, les caractéris-

1. Il n'y a pas de définition standard de la très petite entreprise, ni de paramètres à considérer pour la définir. Après avoir consulté différentes sources, nous avons considéré comme TPE, celles ayant au maximum 10 travailleurs. 
tiques individuelles des chefs d'entreprise, le comportement commercial de l'entreprise, le système d'information, le système d'organisation et la politique commerciale.

En 1988, Aaby et Slater ont réalisé une nouvelle synthèse en résumant et en regroupant les facteurs de ces différents travaux selon trois types, soit les caractéristiques de l'entreprise, leurs compétences et les facteurs en rapport avec leur stratégie.

D'autres études ont permis de définir des typologies générales des petites entreprises à partir non seulement des variables de type quantitatif, mais aussi qualitatif. Signalons celles qui s'arrêtent aux objectifs du chef d'entreprise (Marchesnay, 1988), à la stratégie de la direction (Cooper et Dulkenberg, 1982), ainsi que d'autres concernant le cycle de vie de l'entreprise (Greiner, 1972; Vargas, 1984), et Julien (1990) résume ces typologies en une typologie globale sur un continuum se composant de six éléments: dimension brute, secteur, marché, organisation, stratégies et objectifs, technologie et innovation.

Toutes ces études définissent un cadre théorique permettant de situer l'analyse des PME/PMI. Mais lorsque nous voulons trouver un tel cadre pour l'étude de la très petite entreprise, nous constatons qu'un grand nombre des facteurs et des éléments considérés doivent être adaptés à leurs caractéristiques particulières. Bien souvent, les variables de type structurel vont se situer à une extrémité du continuum (par exemple, le nombre de travailleurs, le niveau d'organisation...) ce qui ne permet pas d'établir de différences significatives entre leurs valeurs extrêmes. Par contre, si l'accent est mis sur les variables touchant les attitudes des entrepreneurs, on pourra trouver une plus large fourchette de possibilités différentielles nous permettant de trouver des différences significatives.

\section{Méthodologie}

Pour notre étude, nous avons utilisé des méthodes tant qualitatives que quantitatives. Pour l'analyse quantitative, nous avons fait une enquête à partir d'un échantillon aléatoire d'entreprises exportatrices de Girona. Pour la qualitative, nous avons interviewé quelques chefs de TPE. Le résumé des variables étudiées et de leur mesure est présenté aux annexes 1 et 2 .

Les premiers résultats montrent que le nombre de petites entreprises exportatrices est d'environ 165 . Notre échantillon comptait 60 entreprises, soit une fiabilité de $95,5 \%$ et une erreur statistique de $\pm 5 \%$. 
Description de l'échantillon: suivantes :

Les caractéristiques générales des 60 entreprises enquêtées sont les

\begin{tabular}{|c|c|c|c|c|c|c|c|}
\hline Secteur & $\begin{array}{l}\text { Alimen- } \\
\text { tation }\end{array}$ & Textile & $\begin{array}{l}\text { Bois, } \\
\text { liège }\end{array}$ & Papier & Chimie & $\begin{array}{l}\text { Métal- } \\
\text { lurgie }\end{array}$ & $\begin{array}{c}\text { Produits } \\
\text { d'artisanat }\end{array}$ \\
\hline $\begin{array}{l}\text { Nombre } \\
\text { d'entreprises }\end{array}$ & 8 & 2 & 13 & 12 & 3 & 16 & 6 \\
\hline \multicolumn{2}{|c|}{ Nombre de travailleurs } & & $0-4$ & & \multicolumn{2}{|l|}{$5-8$} & $8-10$ \\
\hline \multicolumn{3}{|c|}{ Nombre d'entreprises } & 16 & & \multicolumn{2}{|l|}{16} & 28 \\
\hline \multicolumn{2}{|c|}{ Âge de l'entreprise } & $0-5$ ans & \multicolumn{2}{|r|}{$5-15$ ans } & \multicolumn{2}{|c|}{$15-30$ ans } & $>30$ ans \\
\hline \multicolumn{2}{|c|}{ Nombre d'entreprises } & 10 & \multicolumn{2}{|r|}{14} & \multicolumn{2}{|c|}{24} & 12 \\
\hline \multicolumn{2}{|c|}{ Forme juridique } & \multicolumn{2}{|l|}{ IN } & SL & \multicolumn{2}{|c|}{$\mathrm{SA}$} & $\mathrm{A} 1$ \\
\hline \multicolumn{2}{|c|}{ Nombre d'entreprises } & \multicolumn{2}{|l|}{16} & 16 & \multicolumn{2}{|c|}{26} & 2 \\
\hline
\end{tabular}

\section{Les résultats}

\subsection{L'analyse unie et bidimensionnelle des variables}

Les variables ont été analysées une par une, puis ont été croisées deux par deux. Nous commenterons d'abord les relations chi-deux les plus significatives.

Caractéristiques de l'entreprise. Le profil d'une TPE internationalisée dans les cantons de Girona est celui d'une société familiale (71,4\% des cas) se consacrant à l'exportation dans le secteur du bois $(21,6 \%)$, de la métallurgie $(26,6 \%)$, de l'artisanat (20\%) ou de l'alimentation (13,3\%); ses produits sont majoritairement artisanaux $(53,6 \%)$. Par conséquent, le niveau moyen de développement technologique est peu élevé ( $63 \%$ n'ont aucune automatisation), avec un chiffre d'affaires moyen de 125 millions de pesetas dont $27 \%$ pour l'exportation. Elle se consacre à cette activité en moyenne depuis trois ans.

Nous pouvons trouver une association entre le degré de développement technologique des entreprises (nul, moyen et total) et le type de produits fabriqués (artisanaux, de technologie moyenne et de haute technologie) ${ }^{2}$.

2. $\chi^{2}=34,33$ avec 4 degrés de liberté significative pour $a=0,01$. 
Ressources humaines. L'organigramme organisationnel et fonctionnel est très simple et peu spécialisé selon les fonctions. Il y a, en moyenne, sept travailleurs peu qualifiés; près de $67 \%$ des entreprises n'ont aucun travailleur ayant fait des études supérieures. On trouve aussi un rapport entre les années d'études des travailleurs et la branche d'activité de l'entreprise ${ }^{3}$ : la branche de la métallurgie est celle qui présente le niveau de qualification le plus élevé (87,5\% ont des études supérieures), suivie de l'alimentation avec 62,5\%. Quant à la connaissance des langues étrangères, la langue la plus connue est le français, suivie de l'anglais; dans $82,1 \%$ des entreprises un travailleur connaît le français; dans $64,28 \%$, un travailleur connaît l'anglais ; et seulement $25 \%$ des entreprises ont un travailleur connaissant une autre langue. La langue la plus connue après le français et l'anglais est l'allemand.

Internationalisation. Les importations ne constituent pas un vecteur important pour l'internationalisation; $64 \%$ des entreprises ne réalisent aucune importation. Nous allons plutôt nous en tenir à l'exportation pour caractériser l'internationalisation des TPE.

L'exportation se fait majoritairement vers les pays de la $\mathrm{CE}$, avant tout en France (75\% des entreprises), au Portugal $(39,29 \%)$, en Italie $(35,71 \%$ ) et en Allemagne (32,14\%). Seulement $14,29 \%$ des entreprises interrogées exportent vers l'Angleterre, à cause des difficultés de transport. Les États-Unis $(28,57 \%)$ et l'Amérique du Sud $(21,43 \%)$ reçoivent également des produits des TPE. La France a été le pays dans lequel il a été le plus facile de s'introduire, à l'opposé des États-Unis, comme nous avons pu le constater à la suite d'entretiens effectués avec les chefs d'entreprise.

Si nous nous arrêtons maintenant au type de produit exporté vers les différents pays, nous pouvons trouver les relations suivantes: vers les pays les plus développés de la Communauté européenne, nous exportons des produits artisanaux et des produits de haute technologie. Vers le Portugal, nous exportons aussi majoritairement des produits de haute technologie. En Amérique du Nord, c'est surtout des produits artisanaux, alors qu'en Amérique du Sud, les produits exportés sont majoritairement de haute technologie. Le calcul du chi-deux entre les pays et le type d'article produit sont significatifs à différents niveaux, comme nous pouvons le voir au tableau 1 .

Si l'on observe ce tableau, on voit que le rapport entre les différents secteurs d'activité et l'exportation vers la France n'est pas significatif; à cause de la proximité, on y exporte toutes sortes d'articles, comme on l'a dit plus haut, pour des raisons économiques et des ressemblances de goûts. Cet échange a donc clairement un caractère intrabranche.

3. $\chi^{2}=14,08$ avec 6 degrés de liberté significative pour $a=0,02$. 
TABLEAU 1

Relations chi-deux

\begin{tabular}{lccccccccc}
\hline Pays & France & $\begin{array}{c}\text { Angle- } \\
\text { terre }\end{array}$ & $\begin{array}{c}\text { Alle- } \\
\text { magne }\end{array}$ & Italie & Benelux & Portugal $\begin{array}{c}\text { Amé- } \\
\text { rique } \\
\text { du Nord }\end{array}$ & $\begin{array}{c}\text { Amé- } \\
\text { rique } \\
\text { du Sud }\end{array}$ & Secteur \\
\hline Secteur & & 21,78 & 14,78 & 27,68 & 16,83 & & 18,96 & 33,91 & \\
& & $(6)$ & $(6)$ & $(6)$ & $(6)$ & & $(6)$ & $(6)$ & \\
& & $* * * *$ & $*$ & $* * * *$ & $* *$ & & $* * *$ & $* * * *$ & \\
\hline Type & 11,23 & 14,18 & & & 5,89 & 18,77 & 13,69 & 13,63 & 34,33 \\
& $(2)$ & $(2)$ & & & $(2)$ & $(2)$ & $(2)$ & $(2)$ & $(4)$ \\
& $* * *$ & $* * * *$ & & & $*$ & $* * * *$ & $* * * *$ & $* * * *$ & $* *$ \\
\hline
\end{tabular}

* Signification supérieure à $a=0,05$

** Signification supérieure à $a=0,01$

*** Signification supérieure à $a=0,005$

**** Signification supérieure à $a=0,001$

Les valeurs entre parenthèses indiquent les degrés de liberté.

Les exportations des différents secteurs se distribuent de la manière suivante: celui de l'alimentation surtout vers l'Allemagne, celui du bois et du liège vers l'Amérique du Nord et le Benelux, celui de l'artisanat vers le Benelux et l'Amérique du Nord, celui de la chimie vers l'Angleterre, l'Allemagne, l'Amérique du Nord et le Benelux, et celui du métal vers l'Amérique du Sud et le Portugal. Nous constatons également que dans le cas des exportations vers les pays les moins développés, tels que l'Amérique du Sud et le Portugal, le niveau de développement technologique des articles exportés est élevé, alors que vers les pays les plus développés, nous exportons surtout des produits artisanaux.

Si l'on étudie le type d'aides reçues par les différentes entreprises, nous constatons que ce sont normalement des aides de type informationnel, surtout des renseignements touchant les entreprises et les différents secteurs de production, les démarches administratives, les problèmes légaux et la situation économique des différents pays. Il a été très difficile d'obtenir des renseignements sur les types d'aides et de crédits demandés, ainsi que sur les conditions d'obtention. Les TPE les obtiennent, à titre personnel $(45,8 \%)$ ou, en second lieu, en s'adressant aux chambres de commerce $(27 \%)$.

Les plus grandes difficultés rencontrées pour l'exportation sont, d'une part, le manque d'aide et, d'autre part, le taux de change de la peseta. La capacité de production et l'image de nos produits ne sont pas un obstacle pour la plupart des TPE. 
Le marketing le plus utilisé passe par les chambres de commerce $(32,2 \%)$ et par des solutions personnelles $(32,2 \%)$. Seulement $10,7 \%$ des entreprises utilisent les maisons de commerce pour réaliser leurs exportations $\mathrm{s}^{4}$.

Les moyens de transport les plus utilisés pour les exportations sont les voies routières $(85,7 \%)$, suivies des voies maritimes $(39,3 \%)$.

L'encaissement se fait par traite $(42,8 \%)$ ou crédit documentaire $(32,2 \%)$, bien que $25 \%$ déclarent le faire au comptant. La plupart prennent une assurance $(57,1 \%)$, mais $42,9 \%$ soulignent que les difficultés concernant l'encaissement conditionnent leurs exportations.

La participation à des associations professionnelles est très peu courante : $50 \%$ n'appartiennent à aucune association, $25 \%$ appartiennent à des associations locales, et les autres font partie d'associations nationales. Aucune n'appartient à des associations internationales.

Perspectives d'avenir. Plus de la moitié (57\%) sont d'avis que l'avenir de la TPE se présente mal, bien qu'ils espèrent qu'à long terme la situation changera. La plupart sont indifférents à l'entrée dans la $\mathrm{CE}$, bien que la moitié considèrent que cette entrée peut être positive pour les exportations.

Après avoir expliqué les variables de manière individuelle et avoir analysé quelques rapports significatifs en les comparant deux par deux, nous allons effectuer une analyse factorielle pour pouvoir étudier plus à fond les rapports existants et pour synthétiser tous ces rapports.

\subsection{L'analyse factorielle des correspondances multiples (ACM)}

Vu le nombre important d'éléments, il est nécessaire de réaliser une analyse à correspondances multiples (ACM) afin de résumer les informations obtenues grâce aux données.

L'analyse des résultats de l'ACM indique que les trois premiers axes sont ceux qui contribuent le plus à l'explication des différentes variables contenues dans l'étude, avec des poids de $15,01 \%, 11,72 \%$ et $11,20 \%$, c'est-à-dire un total accumulé de $37,93 \%$. Ces axes sont présentés aux graphiques 1 et 2 .

4. On trouve un rapport significatif $\chi^{2}=11,35$ avec 5 degrés de liberté $a=0,05$ entre le type de marketing utilisé et le niveau d'études des travailleurs. 
GRAPHIQUE 1

Projection des entreprises sur les axes 1 et 2

AXE 2

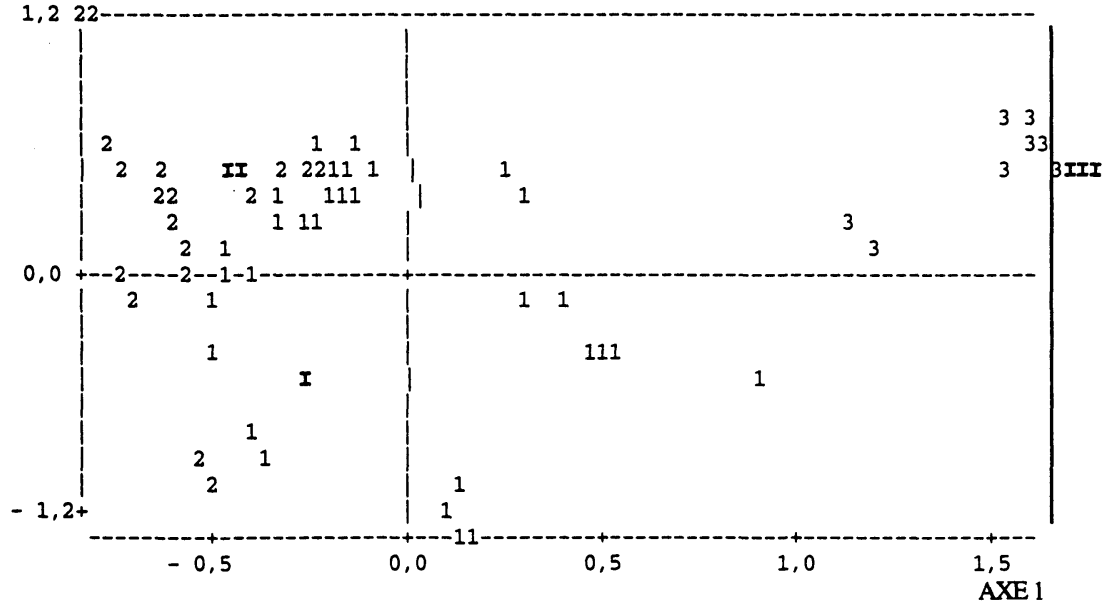

GRAPHIQUE 2

Projection des entreprises sur les axes 1 et 3

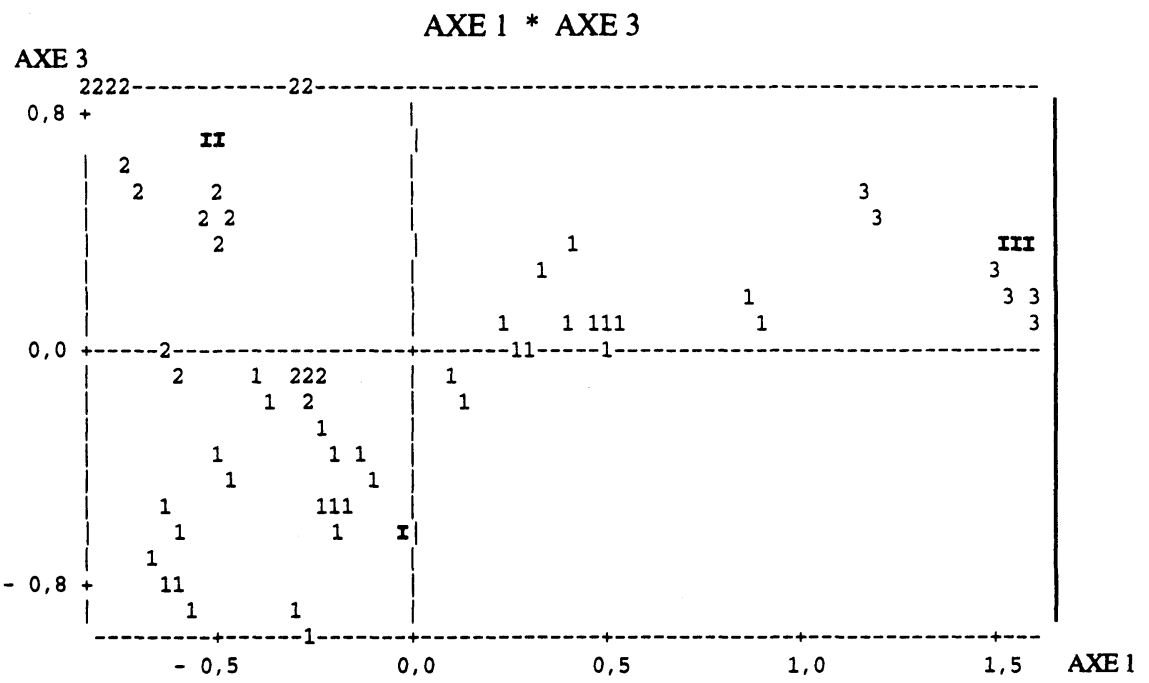


Premier axe factoriel. Cet axe montre significativement à droite le secteur artisanal (PAP), avec des niveaux d'ancienneté importants qui vont de 15 à 30 ans (An4, Ea4), un niveau technologique rudimentaire (NUL), une préoccupation par rapport au manque d'aide (Ea5), une faible sensibilité à certains problèmes tels que la valeur de la monnaie et les difficultés rencontrées lorsque nous voulons connaître les marchés (E\$1, De1), l'utilisation de ressources en marketing publicitaire (PUB), des ressources humaines peu qualifiées (Es1) et un niveau d'exportation peu élevé (BAIX). De l'autre côté de l'axe, nous voyons apparaître le secteur du métal (MET), les variables indiquant un degré élevé de développement technologique (AT, TOT), une ancienneté allant de 5 à 10 ans (An2, Ea2), une préoccupation par rapport à la situation géographique, à l'image de marque du pays et aux démarches administratives et légales (Ep5, Eg4, Dl5, Da5), une attitude optimiste quant à l'obtention d'un financement, ainsi que par rapport à la crise actuelle les affectant (Dc2, Lla) et un niveau d'exportation élevé (ALT).

Deuxième axe. Cet axe montre, en bas, à l'échelle négative, le secteur de l'alimentation (ALI), avec une ancienneté allant de 1 à 5 ans (An1, Ae1) et des ressources en marketing démontrant une attitude dynamique (VIA, FIR), alors que de l'autre côté de l'axe, nous voyons clairement apparaître les variables indiquant un niveau de développement technologique et d'innovation élevé (AT, TOT), des attitudes peu pessimistes par rapport à la crise actuelle (Lla) et une bonne connaissance des marchés (Del).

Troisième axe. Cet axe projette significativement du côté positif (en haut à gauche) le secteur du textile (TEX), avec une ancienneté allant de 6 à 15 ans (An2, Ea2), un niveau de développement technologique élevé (TOT, AT), une préoccupation concernant la situation géographique $(\mathrm{Eg} 4)$, une attitude peu pessimiste par rapport à la crise actuelle (Lla) et des ressources en marketing très élevées (FIR, CAP). Dans la partie négative de l'axe, nous voyons apparaître le secteur du bois et du liège (FIS), avec une ancienneté plus élevée, allant de 15 à 30 ans (Ae3, An3), un développement technologique moyen (PAR), un recours à des ressources de marketing diverses (VIA, PER), une préoccupation par rapport aux difficultés de type légal et de connaissance des marchés (De5, D14), ainsi qu'un certain optimisme concernant la résolution de la crise actuelle et de l'entrée dans la CE (Mom, P1).

L'ACM fait apparaître des regroupements qui suggèrent différentes typologies d'entreprises. Nous pouvons constater, d'abord, qu'il est difficile de donner un nom ou de caractériser clairement les différents axes expliqués, ce qui confirme que les typologies différentielles des très petites entreprises apparaissent souvent d'une façon dispersée. La classification ascendante hiérarchique nous permettra de définir différents types d'entreprises. 


\section{Les typologies des TPE exportatrices}

\subsection{Classification ascendante hiérarchique (CAH)}

Après avoir fait plusieurs $\mathrm{CAH}$ avec trois types et avec cinq types, nous avons constaté qu'il y a un type d'entreprises qui se différencie, car les facteurs qui interviennent dans l'analyse se modifient constamment, alors que le reste est plus ambigu et que les entreprises sont classées différemment. La classification que nous allons utiliser pour expliquer les différentes typologies est une classification en trois classes sur les axes factoriels calculés au cours de l'ACM antérieur et sur lesquelles interviennent les 46 variables de l'analyse, afin de ne pas perdre d'information.

Pour ces trois classes, nous rencontrons une inertie totale de 2,42 qui se distribue de la façon suivante: une inertie interclasses de 0,874 et une inertie intraclasses de $0,94,0,65$ et 0,08 , respectivement. Nous voyons donc que la première et la deuxième classes sont absolument dispersées et qu'il est difficile de distinguer les typologies de chacune de ces classes.

\section{2. Étude des typologies}

\section{TYPE 1 : Entreprises qui se laissent influencer par leur environnement}

Ce sont des entreprises peu anciennes $(58,82 \%$ ont entre 15 et 30 ans) majoritairement dans le secteur du bois et du liège $(38,24 \%)$ ou de l'alimentation $(23,53 \%)$, avec un degré de technologie moyen et possédant une vision peu optimiste en ce qui a trait aux difficultés qui peuvent se présenter. La moitié d'entre elles n'ont aucun employé possédant des études supérieures et une seule n'a aucun employé possédant une connaissance de langue étrangère. La moitié des employés connaissant une langue étrangère connaissent le français et l'autre moitié l'anglais. Près de $45 \%$ des entreprises sont des sociétés anonymes ou à responsabilité limitée alors que le reste correspond à des entreprises de type individuel. Le niveau d'exportation est moyen et les variables qui touchent l'attitude stratégique du chef d'entreprise par rapport à l'exportation ont des valeurs moyennes sur le continuum défini pour chacune d'entre elles. Afin de pénétrer sur les marchés étrangers, elles utilisent habituellement les ressources de marketing que les chambres de commerce mettent à leur disposition : participation commune d'entreprises à des foires internationales et organisation de voyages de prise de contact avec les entreprises étrangères.

Nous pouvons constater, dans la représentation des classes sur les axes factoriels 1 et 2 (graphique 1), qu'elles forment une classe très dispersée qui 
recouvre la zone inférieure du second axe et la partie centrale et gauche du premier axe.

En examinant la représentation sur les axes 1 et 3 (graphique 2), nous constatons à nouveau la dispersion sur l'axe 1 , mais nous remarquons une concentration surtout sur la partie inférieure du troisième axe.

\section{TYPE 2: Entreprises qui interviennent sur leur environnement}

Ce sont des entreprises d'une ancienneté surtout moyenne, qui depuis déjà quelques années exportent dans le secteur du métal $(88,9 \%)$, de haute technologie. Elles disposent au moins de quelques employés possédant des études supérieures et une connaissance en langue étrangère. Toutes ces entreprises sont des entreprises anonymes. Leur niveau d'exportation est élevé. Ces entreprises n'ont pas de difficultés pour obtenir de l'information ainsi que différents types d'aides. La situation par rapport à la crise ne les préoccupe qu'à long terme et la situation géographique de l'Espagne représente un inconvénient pour elles, puisque leurs exportations se dirigent dans $85 \%$ des cas vers les pays de l'Amérique du Sud. L'attitude stratégique du chef d'entreprise est fondamentalement positive face aux difficultés inhérentes à l'exportation et quant au futur proche. Dix-huit des soixante entreprises interrogées répondent à cette typologie.

Dans la représentation graphique des entreprises de ce deuxième type sur les axes factoriels 1 et 2 (graphique 1), nous constatons que bien qu'elles présentent un niveau de similarité élevé avec celles de la première classe, elles s'en distinguent en se situant dans la partie la plus à gauche du premier axe et sur la partie la plus supérieure du deuxième axe. La projection sur les axes 1 et 3 du graphique 2 confirme les projections de l'axe 1 et nous voyons qu'elles se situent dans la partie supérieure du troisième axe caractérisé par certaines variables indiquant un niveau de développement élevé.

\section{TYPE 3: Entreprises traditionnelles}

Ce sont des entreprises très anciennes (100\%) avec plus de 30 ans d'activité, de type artisanal $(100 \%)$ et se caractérisant par un degré élevé de difficultés pour résoudre les problèmes inhérents à l'exportation. Nous remarquons aussi que $45 \%$ d'entre elles n'ont aucun employé possédant des études supérieures. La majorité possèdent quelques employés avec une connaissance des langues étrangères, surtout le français. Enfin, $75 \%$ d'entre elles sont des sociétés et $25 \%$ sont des entreprises individuelles. Elles ont en général beaucoup de difficultés à obtenir de l'aide et se montrent peu sensibles à la variation du taux de change. Le niveau d'exportation de ces entreprises est faible; huit entreprises interrogées répondent à cette typologie. 
La projection sur les axes 1 et 2 (graphique 1), situe ces entreprises anciennes dans la partie droite du premier axe et elles ne présentent aucune similarité avec les types antérieurs ; elles forment donc une classe assez clairement différenciée. Dans la projection sur les axes 1 et 3 (graphique 2), nous voyons qu'il en est de même, c'est-à-dire que ce qui les caractérise est fondamentalement la partie positive du premier axe factoriel défini.

\section{Conclusion}

Ce sont les attitudes stratégiques du chef d'entreprise qui, avec les quelques caractéristiques générales ou structurelles de l'entreprise (secteur), font qu'une entreprise a un comportement international plus ou moins fort. Un chef d'entreprise décidé, capable d'obtenir l'information dont il a besoin est prêt à prendre des risques et est optimiste, c'est-à-dire qu'il est doté d'un esprit entrepreneurial qui le dispose à surmonter les obstacles et explique en définitive pourquoi son entreprise exporte de façon importante.

La capacité de production, compte tenu de la petite taille de l'entreprise et, par conséquent, de l'impossibilité d'obtenir des économies d'échelles, n'est pas perçue par les chefs d'entreprises comme un obstacle pour s'introduire sur les marchés internationaux. Ces économies d'échelles ont beaucoup retenu l'attention de la littérature théorique; mais de nombreuses études empiriques ont trouvé très peu ou aucun rapport entre la dimension de l'usine et la productivité. Ce qui est le plus important pour la productivité ou les coûts unitaires, c'est que la production soit organisée dans l'usine (Daly, Keyes et Spence, 1968; Wonnacott et Wonnacott, 1967 ; Verdoorn, 1960). Il faut considérer également que même si les coûts variables peuvent être supérieurs pour les petites entreprises, les coûts fixes sont beaucoup plus importants pour les grandes, ce qui peut compenser et permettre à la TPE d'obtenir des coûts unitaires compétitifs.

Les conditions macro-économiques dans lesquelles baigne l'entreprise ont une importance cruciale au regard des possibilités d'exportation. Ainsi, le taux de change de la peseta par rapport aux autres monnaies européennes ou au dollar conditionne dans une large mesure les exportations. Dernièrement, en Espagne, il y a eu deux dévaluations qui ont placé les entreprises de Girona dans une position beaucoup plus favorable.

Nous pouvons considérer aussi que la crise économique mondiale affecte les exportations, car la demande de biens hautement spécialisés que peuvent exporter les TPE diminue fortement lorsque les revenus baissent, puisque la demande pour ce type de biens est fortement corrélée avec le niveau de revenu. 
Dans la discussion théorique, nous observions déjà qu'il est possible de faire deux types d'analyses en ce qui concerne l'internationalisation: celles qui touchent la décision de s'introduire ou non sur les marchés étrangers et celles qui étudient la performance dans l'exportation. Notre travail se situe dans le deuxième groupe. Vu le faible taux de très petites entreprises exportatrices dans notre région, 165 en tout sur un ensemble de 11851 TPE, il faudrait réaliser un travail complémentaire qui préciserait les caractéristiques distinctives des entreprises exportatrices par rapport à celles qui n'exportent pas, afin de compléter le tableau de la situation de la TPE géronaise.

De toute façon, même en se situant dans le petit monde des très petites entreprises exportatrices, de celles qui ont réussi à s'introduire sur les marchés internationaux, nous pouvons distinguer plusieurs types différents et même relier ces types à divers niveaux de performance.

\section{Bibliographie}

AABY, N. et S. SLATER (1988), «Management influence on export performance: a review of the empirical literature 1978-88», International Marketing Review, vol. $6, n^{\circ} 4$, p. 7-26.

BADOUX, M. et A. RoNCIN (1980), «Évaluation et analyse des performances à l'exportation des petites et moyennes entreprises industrielles », Rapport Cordes, Paris, p. 25-78.

BaLDwin, R. (1989), «The growth effects of $1992 »$, Economic Policy, octobre, $\mathrm{n}^{\circ} 9$.

BENOIT, C. et M.D. RousSEAU (1990), «La gestion des ressources humaines dans les petites et moyennes entreprises au Québec», Revue Internationale PME, vol. $3, n^{\circ} 1$, p. $40-55$.

BILKEY, W.J. (1978), «An attempted integration of literature on export behaviour of firms », Journal of International Business Studies, été, p. 33-46.

BONET, E. (1988), Introducción al acta Unica Europea, Barcelona, Publicaciones de la Escuela Superior de Gestión Comercial y Marketing.

CÁmara Oficial de Comercio e Industria De Girona (1981), Girona exporta, Girona.

CONEJos, J. et al. (1991), Dades bàsiques de l'estructura industrial de Catalunya, Barcelona, Generalitat de Catalunya.

COOPER, A. et C. DUNNLKELBERG (1982), «Entrepreneurial typologies: an empirical study », Frontiers of Entrepreneurship Research, Babson College. 
Daly, D.J., B.A. KeYes et E.J. SPENCE (1968), Scale and Specialization in Canadian Manufacturing, Ottawa, Conseil économique Canada.

FERRER, F. (1972), L'estructura comercial a les comarques gironines, Girona, Camara Oficial de Comerç i Industria.

GARNIER, G. (1982), "Comparative export behaviour of small Canadian firms in the printing in electrical industries », dans Export Management: An International Context, New York, Praeger Publishers, p. 113-131.

GioRdano, Y. (1984), «Stratégie d'entreprise et décision d'exporter », Revue Française de Gestion, juin-août, p. 113-119.

GRANELL, F. (1984), La exportación y los mercados internacionales, Barcelona, Editorial Hispano Europea, S.A.

GREENAWAY, D. (1988), «Intra-industry trade, intra-firm trade and european integration: evidence, gains and policy aspects », dans J. Dunning et P. Robson (éd.), Multinationals and the European Community, Oxford et New York, p. 51-70.

GRENIER, L. (1972), «Evolution and revolution of organizations growth », Harvard Business Review, vol. 50, $\mathrm{n}^{\circ} 4$, p. 37-46.

GuAL, J. (1991), La industria catalana en el anys 90, Barcelona, Ed. Ariel.

Higler, M.T. et R.J. Hoover (1989), «Dynamics of the export decision process », Journal of Global Marketing, vol. 2, n 3, p. 71-93.

JUlien, P.A. (1990), «Vers une typologie multi-critère de la PME », Revue Internationale $P M E$, vol. $3, \mathrm{n}^{\circ} 3-4$, p. 411-427.

Julien, P.A. et M. MARChESNAY (éd.) (1988), La petite entreprise, Paris, Vuibert gestion.

LINDER, S.B. (1961), An Essay on Trade and Transformation, New York, John Wiley and Sons.

LOERTSCHER, R.Y. et F. WOLTER (1980), «Determinants of intra-industry trade : among countries and across industries », Weltwirtschaftliches Archiv, p. 281-293.

PORTER, M. (1982), Choix Stratégie et Concurrence, Paris, Économica.

PRAS, B. et E. RouX (1985), «Forces et faiblesses des dirigeants de PMI », Enseignement et gestion, $\mathrm{n}^{\circ} 36$, p. 59-65.

REFFAIT, P. et E. RouX (1981), « Le profil idéal de la PME exportatrice», Revue Française de Gestion, janvier-février, p. 88-96.

REID, S.D. (1981), «The decision maker and export entry and expansion », Journal of International Business Studies, vol. 12, p. 101-112. 
Roux, E. (1986), «Les modèles intégrés de la décision d'exporter en PME/PMI », Recherche et Applications en Management, $n^{\circ} 3$, p. 27-42.

Roux, E. (1991), Les facteurs explicatifs de la décision d'exporter en PMI: rôle de l'attitude du dirigeant envers le risque, Thèse d'État en sciences de gestion, IAE d'Aix-en-Provence.

VARGAS, G. (1984), "Les crises de croissance de la PMI/PME», Revue Francaise de Gestion, janvier-février.

VERDOON, P.J. (1960), The Intra-block Trade of Benelux, Robinson (éd.), p. 291-329.

Wiedersheim-Paul, F., H. Olson et L. WelCh (1978), «Pre-export activity: the first step in internationalisation », Journal of International Business Studies, printemps-été, vol. $9, \mathrm{n}^{\circ} 1$, p. 47-56.

WonnacotT, R.J. et P. WonnacotT (1967), Free Trade Between the United States and Canada: The Potential Economics Effects, Cambridge, Mass., Harvard University Press. 


\section{ANNEXE 1 \\ Définition des variables et modalités}

Secteur d'activité : BRANCA
ALI : Alimentation
TEX : Textile
FIS : Bois et liège
PAP : Céramique, autres
QUI : Chimie
MET : Métallurgie
AL2 : Autres
Forme juridique :
TEMPRESA
IN : Individuelle
SL : S. Limitée
SA : S. anonyme
A1 : Autres

$\begin{array}{ll}\text { Exportation France : FRAN } & \text { Type d'Aide : TAJUT } \\ \text { Fs : Oui } & \text { NO : Aucune } \\ \text { Fn : Non } & \text { INF : Informative } \\ & \text { LEG : :Légale } \\ \text { Export. Allemagne : ANGL } & \text { FIN : Financière } \\ \text { Als : Oui } & \text { A25: Autres }\end{array}$

Ann: Non

Export. Allemagne : ALEM

Als : Oui

Aln : Non

Export. Portugal : PORTU

Ps : Oui

Pn : Non
Marketing : MARK
CAP : Aucune
COC : Chambre de Comm.
PER : Personnel
PUB : Publicité
FIR : Foires
VIA : Voyages

Nombre de travailleurs : NTREB

Exportation Italie : ITAL

Nt1 : Moins de 5

Is : Oui

In : Non

Méthodes d'exportation : COMEX

DIR : Directement

Nt2: De 5 à 8

Nt3 : Plus de 8

CTR : Maison de commerce

A27 : Autres

Type d'article : TARTICLE

AR : Artisanal

Exportation BENELUX : BENEL Obtient l'information nécessaire

Bs : Oui

TM : Techno. moyenne

AT : Haute technologie

Bn : Non

personnellement : INFCP

Ips : Oui

Ipn : Non

Ancienneté Entrepr. : ANT

An1: De 1 à 5 ans

An2 : De 6-15

An3 : De 16-30

An4 : Plus de 30

Exportation Amérique du Nord: Ancienneté export : ANTEXP

NORD

Ns : Oui

Ael : De 1 à 5 ans

Ns : Non

Ae2 : De 6-15

Ae3 : De 16-30

Ae4 : Plus de 30

Obtient l'information nécessaire Chambre de comm.: INFCC

Exportation Amérique du Sud : SUD

Ics : Oui

Ss : Oui

Obtient l'information nécessaire

Icn : Non

Sn : Non

des maisons de commerce : INFCT

Its : Oui

Itn : Non

Niveau d'études travail.: ES

Assurances : ASSEG

Es 1: Aucun avec des études

supérieures

Es2 : 1 ou plus

SEM : Toujours

Obtient l'information nécessaire des bureaux commerciaux :

VEG : Parfois

INFOC

MAI : Jamais

Ios : Oui

Ion : Non

Difficulté pour obtenir

Difficulté pour obtenir

Difficulté pour obtenir information information sur marchés: VALD1 information sur secteur: VALD2 sur démarches adm. : VALD3

Dp1 : Aucune

De1 : Aucune

Da1 : Aucune

Dp2 : Un peu

De2: Un peu

Da2 : Un peu

Dp3 : Moyenne

De3 : Moyenne

Da3 : Moyenne

Dp4 : Importante

De4 : Importante

Da4 : Importante

Dp5 : Beaucoup

De5 : Beaucoup 


\section{ANNEXe 1 (suite) \\ Définition des variables et modalités}

\begin{tabular}{|c|c|c|}
\hline $\begin{array}{l}\text { Difficulté pour obtenir } \\
\text { information } \\
\text { sur sujets légaux : VALD4 }\end{array}$ & $\begin{array}{l}\text { Difficulté pour obtenir } \\
\text { information sur financement : } \\
\text { VALD5 }\end{array}$ & $\begin{array}{l}\text { Importance du type de } \\
\text { change : VALE1 }\end{array}$ \\
\hline D11 : Aucune & Dc1 : Aucune & E\$1: Aucune \\
\hline D12: Un peu & Dc2 : Un peu & E\$2: Un peu \\
\hline D13 : Moyenne & Dc3 : Moyenne & E\$3: Moyenne \\
\hline D14 : Importante & Dc4 : Importante & $\mathrm{E} \$ 4$ : Importante \\
\hline D15 : Beaucoup & Dc5 : Beaucoup & E\$5 : Beaucoup \\
\hline $\begin{array}{l}\text { Importance de l'image de } \\
\text { notre pays : VALE2 }\end{array}$ & $\begin{array}{l}\text { Importance de l'image de } \\
\text { nos produits : VALE3 }\end{array}$ & $\begin{array}{l}\text { Importance du manque } \\
\text { d'aide : VALE4 }\end{array}$ \\
\hline Epl : Aucune & Eprl : Aucune & Eal : Aucune \\
\hline Ep2: Un peu & Epr2: Un peu & Ea2: Un peu \\
\hline Ep3 : Moyenne & Epr3 : Moyenne & Ea3 : Moyenne \\
\hline Ep4 : Importante & Epr4 : Importante & Ea4: Importante \\
\hline Ep5 : Beaucoup & Epr5 : Beaucoup & Ea5 : Beaucoup \\
\hline $\begin{array}{l}\text { Importance de la capacité } \\
\text { de production : VALE5 } \\
\text { Ec1 : Aucune } \\
\text { Ec2 : Un peu } \\
\text { Ec3 : Moyenne } \\
\text { Ec4 : Importante } \\
\text { Ec5: Beaucoup }\end{array}$ & $\begin{array}{l}\text { Effet de l'incorporation } \\
\text { au marché unique sur } \\
\text { l'engagement de personnel : } \\
\text { VALC1 } \\
\text { P1 : Positif } \\
\text { P2 : Négatif } \\
\text { P3 : Indifférent }\end{array}$ & $\begin{array}{l}\text { Effet de l'incorporation } \\
\text { au marché unique sur } \\
\text { les exportations : } \\
\text { VALC2 } \\
\text { Ex1 : Position } \\
\text { Ex2: Négatif } \\
\text { Ex3 : Indifférent }\end{array}$ \\
\hline $\begin{array}{l}\text { Importance de la situation } \\
\text { géographique : VALE6 } \\
\text { Eg1 : Aucune } \\
\text { Eg2: Un peu } \\
\text { Eg3 : Moyenne } \\
\text { Eg4: Importante } \\
\text { Eg5: Beaucoup }\end{array}$ & $\begin{array}{l}\text { Niveau de rapport avec } \\
\text { l'environnement : ASSOC } \\
\text { No : Aucun } \\
\text { Loc : Locaux } \\
\text { Nac : Nationaux }\end{array}$ & $\begin{array}{l}\text { Niveau d'organisation de } \\
\text { l'entreprise : ORGA } \\
\text { Or1 : Sans structure } \\
\text { Or2 }: \text { Structure simple } \\
\text { Or3 : Structurée en départements }\end{array}$ \\
\hline $\begin{array}{l}\text { Systèmes } \\
\text { d'encaissement : SCOBR } \\
\text { COM : Comptant } \\
\text { GIR : Virement } \\
\text { LER : Lettre de change } \\
\text { CDO : Crédit documentaire }\end{array}$ & $\begin{array}{l}\text { Comment elle est affectée } \\
\text { par la crise : AFECCCRIS } \\
\text { Poc : Pas beaucoup } \\
\text { Mom : Momentanément } \\
\text { Cur : À court terme } \\
\text { Lla : À long terme }\end{array}$ & $\begin{array}{l}\text { Chiffre d'affaires: VOLUM } \\
\text { V1 : Moins de } 50 \text { millions } \\
\text { V2: De } 50 \text { à } 100 \\
\text { V3 : Plus de } 100\end{array}$ \\
\hline $\begin{array}{l}\text { Connaissances d'anglais: } \mathrm{CA} \\
\mathrm{Ca} 1: \text { Aucun travailleur } \\
\mathrm{Ca} 2 \text { : Certains travailleurs }\end{array}$ & $\begin{array}{l}\text { Niveau d'exportation : } \\
\text { EXPORT } \\
\text { BAX : Faible }(<25 \%) \\
\text { MIG : Moyen }(25-50 \%) \\
\text { ALT : Élevé (Plus de } 50 \%)\end{array}$ & $\begin{array}{l}\text { Niveau de développement } \\
\text { technologique : AUTOM } \\
\text { TOT : Haut niveau } \\
\text { PAR : Niveau moyen } \\
\text { NUL : Nul }\end{array}$ \\
\hline $\begin{array}{l}\text { Connaissances de français: CF } \\
\text { Cf1: Aucun travailleur } \\
\text { Cf2: Certains travailleurs }\end{array}$ & $\begin{array}{l}\text { Acceptation d'un risque } \\
\text { financier: COBR } \\
\text { Cos : Oui } \\
\text { Con : Non }\end{array}$ & \\
\hline
\end{tabular}

Revue internationale P.M.E., vol. 7, no 2, 1994 
ANNEXE 2

Variables et codes

\begin{tabular}{|c|c|c|}
\hline VARIABLES & CODES & MODALITÉS \\
\hline \multicolumn{3}{|l|}{ 1. Caractères généraux de l'entreprise } \\
\hline Secteur & BRANCA & $1,2,3,4,5,6,7$ \\
\hline Âge de l'entreprise & ANT & $1,2,3,4$ \\
\hline Forme juridique & TEMPRESA & $1,2,3,4$ \\
\hline Type d'article & TARTICLE & $1,2,3$ \\
\hline Niveau de dév. technol. & AUTOM & $1,2,3$ \\
\hline \multicolumn{3}{|l|}{ 2. Ressources humaines } \\
\hline Nombre de travailleurs & NTREB & $1,2,3$ \\
\hline Niveau d'études & ES & 1,2 \\
\hline Connaissance de français & $\mathrm{CF}$ & 1,2 \\
\hline Connaissance d'anglais & $\mathrm{CA}$ & 1,2 \\
\hline Niveau d'organisation & ORGA & $1,2,3$ \\
\hline \multicolumn{3}{|l|}{ 3. Niveau de performance } \\
\hline Niveau de production & VOLUM & $1,2,3$ \\
\hline Niveau d'exportation & EXPORT & $1,2,3$ \\
\hline Ant. exportation & ANTEX & $1,2,3,4$ \\
\hline \multicolumn{3}{|l|}{ 4. Marché pour l'exportation } \\
\hline France & FRAN & 1,2 \\
\hline Angleterre & ANGL & 1,2 \\
\hline Allemagne & ALEM & 1,2 \\
\hline Portugal & PORTU & 1,2 \\
\hline Italie & ITAL & 1,2 \\
\hline Benelux & BENEL & 1,2 \\
\hline Amérique du nord & NORD & 1,2 \\
\hline Amérique du sud & SUD & 1,2 \\
\hline \multicolumn{3}{|l|}{$\begin{array}{l}\text { 5. Attitude stratégique du chef d'entreprise } \\
\text { concernant l'exportation }\end{array}$} \\
\hline Marketing & MARK & $1,2,3,4,5,6$ \\
\hline Méthodes d'exportation & COMEX & $1,2,3$ \\
\hline Obtention inf. personnelle & INFCP & 1,2 \\
\hline Obtention inf. de cham. comm. & INFCC & 1,2 \\
\hline Obtention inf. de maisons de commerce & INFCT & 1,2 \\
\hline Obtention inf. bureaux commerc. & INFOC & 1,2 \\
\hline Type d'aides obtenues & TAJUT & $1,2,3,4,5$ \\
\hline Systèmes d'encaissement & SCOBR & $1,2,3,4$ \\
\hline \multirow{2}{*}{\multicolumn{3}{|c|}{ Ev valuation importance: }} \\
\hline & & \\
\hline Type de change peseta & VALE1 & $1,2,3,4,5$ \\
\hline Image de notre pays & VALE2 & $1,2,3,4,5$ \\
\hline Image de nos produits & VALE3 & $1,2,3,4,5$ \\
\hline Manque d'aide & VALE4 & $1,2,3,4,5$ \\
\hline Capacité de production & VALE5 & $1,2,3,4,5$ \\
\hline Situation géographique & VALE6 & $1,2,3,4,5$ \\
\hline \multicolumn{3}{|c|}{ Éval. de la difficulté d'obtention d'information sur: } \\
\hline Pays & VALD1 & $1,2,3,4,5$ \\
\hline Entreprises du secteur & VALD2 & $1,2,3,4,5$ \\
\hline Démarches administratives & VALD3 & $1,2,3,4,5$ \\
\hline Démarches légales & VALD4 & $1,2,3,4,5$ \\
\hline Information crédits & VALD5 & $1,2,3,4,5$ \\
\hline Comment la crise l'affecte & AFECCRIS & $1,2,3,4$ \\
\hline Effet entrée CE/Personnel & VALC1 & $1,2,3$ \\
\hline Effet entrée CE/Expor & VALC2 & $1,2,3$ \\
\hline Assurances & ASSEG & $1,2,3$ \\
\hline Acception de risque financier & COBR & 1,2 \\
\hline
\end{tabular}

